\title{
Esferocitosis hereditaria. Revisión. Parte I. Historia, demografía, etiopatogenia y diagnóstico
}

\author{
Hereditary spherocytosis. Review. \\ Part I. History, demographics, pathogenesis, and diagnosis
}

\author{
Comité Nacional de Hematología: Dr. Hugo Donato ${ }^{a, b}$, Dra. Renée Leonor Crisp ${ }^{b, c, d}$, \\ Dra. María Cristina Rapetti ${ }^{a}$ Dra. Eliana García y Dra. Myriam Attief
}

\begin{abstract}
RESUMEN
La esferocitosis hereditaria es la anemia hereditaria más frecuente en nuestro país luego de la talasemia menor. En este artículo, se revisan aspectos históricos, demográficos, genéticos y etiopatogénicos dela enfermedad, y se describen las pruebas delaboratorio para su diagnóstico. Se remarca el comportamiento de la enfermedad en nuestra población y se detallan las deficiencias proteicas predominantes en nuestro país. Se enfatiza sobre las nuevas técnicas de laboratorio actualmente disponibles, con alta sensibilidad y especificidad, que permiten realizar un diagnóstico más temprano con volúmenes de muestra mucho menores que los necesarios para las pruebas convencionales.

Palabras clave: esferocitosis hereditaria, fragilidad osmótica, citometría de flujo, anemia hemolítica, hiperbilirrubinemia.
\end{abstract}

\begin{abstract}
Hereditary spherocytosis is the most frequent hereditary anemia excluding beta thalassemia in Argentina. Historical, demographic, genetic and pathogenic aspects of the disease are reviewed, and confirmatory laboratory tests are described.Special characteristics on the outcome of the disease in our population and prevalent protein deficiencies in our country are described. Emphasis is given on new available laboratory tests, which allow an earlier diagnosis using volume of blood samples significantly smaller than required for conventional tests.

Key words: hereditary spherocytosis, osmotic fragility, flow cytometry, hemolytic anemia, hyperbilirubinemia.
\end{abstract}

http:/ /dx.doi.org/10.5546/aap.2015.69

\section{INTRODUCCIÓN}

La esferocitosis hereditaria (ESH) es una anemia hemolítica de observación frecuente, en la cual defectos cuali- o cuantitativos de algunas proteínas de la membrana eritrocitaria (espectrina, ankirina, proteína 4.2, banda 3) llevan a la formación de hematíes de forma esférica, osmóticamente frágiles, que son selectivamente atrapados y destruidos en el bazo. En nuestro país, es la anemia hereditaria más frecuente después de la beta talasemia heterocigota.

\section{HISTORIA}

El concepto de la destrucción prematura de eritrocitos como un proceso patológico fue sugerido por primera vez en 1871 por Vanlair y Masius al describir una paciente con ictericia y marcada esplenomegalia, sin hepatomegalia, que presentaba ataques recurrentes de dolor agudo en abdomen superior. La madre y la hermana presentaban sintomatología similar. Si bien los autores no mencionaron la anemia ni plantearon el concepto de hemólisis como un proceso patológico, el valor de esta publicación residió en la descripción de la presencia de pequeños glóbulos rojos esféricos, que denominaron microcitos. ${ }^{1}$ Aunque postularon dos mecanismos diferentes para la ictericia clínica (el de origen hepático y el de origen sanguíneo), no se le prestó la atención merecida y, durante casi 30 años, la ictericia se asoció a enfermedad hepática. Hayem, en 1898, y Minkowski, en 1900 , mostraron que la ictericia asociada a la anemia hemolítica era diferente a la secundaria a procesos hepáticos. Hayem no la describió como anemia hemolítica, sino como "ictericia esplenomegálica infecciosa crónica". Fue Minkowski quien asoció 
la anemia con la urobilinuria y la esplenomegalia, y postuló que la destrucción de los eritrocitos era atribuible a lesiones en el bazo. De esta manera, a Minkowski se le atribuye el haber reconocido la ictericia como un proceso asociado a la anemia hemolítica. ${ }^{1}$ En la primera década del siglo XX, Chauffard describió la prueba de fragilidad osmótica, que le permitió observar que los eritrocitos de pacientes con ictericia congénita hemolizaban en soluciones salinas hipotónicas, mientras que los eritrocitos de individuos normales no lo hacían. Las observaciones de ambos autores llevaron a que, posteriormente y durante muchos años, la ESH fuera denominada como "enfermedad de Minkowski-Chauffard".

\section{ASPECTOS EPIDEMIOLÓGICOS}

La ESH es la anemia hemolítica hereditaria más frecuente en el mundo. ${ }^{2} \mathrm{Si}$ bien se presenta en todos los grupos étnicos y raciales, es particularmente frecuente en Europa del Norte, con una prevalencia de 1 en 5000 personas. $^{3}$ Sin embargo, dada la frecuencia de formas muy leves de enfermedad que solo se pueden detectar a través de pruebas de laboratorio muy sensibles, se estima que esta prevalencia probablemente sea 4-5 veces mayor. ${ }^{4,5}$ En Estados Unidos, se ha informado que afecta a 1 de cada 2500 personas. ${ }^{2}$ No hay estimaciones confiables en otras poblaciones, pero su frecuencia parece ser especialmente baja en africanos y en personas del sudeste asiático. ${ }^{6}$ En nuestro país, tampoco existen estimaciones de prevalencia, pero es la anemia hemolítica hereditaria más frecuentemente diagnosticada.

\section{TRANSMISIÓN GENÉTICA}

La ESH es transmitida en forma autosómica dominante o recesiva. La más frecuente, que se observa en aproximadamente $75 \%$ de las familias afectadas, es la autosómica dominante..$^{6-8}$ En un estudio multicéntrico sobre 143 casos realizado en nuestro país, la incidencia de formas dominantes fue de $84 \% .{ }^{9}$ Con la excepción de muy aisladas comunicaciones, casi no hay casos conocidos de homocigotas, por lo que se supone que esta situación es incompatible con la vida. En el restante $20-25 \%$ de los casos, ningún padre presenta evidencia hematológica de la enfermedad, y las pruebas de laboratorio confirmatorias son normales o solo presentan alteraciones mínimas, ${ }^{8,10}$ son las llamadas formas recesivas o no-dominantes. Si bien se supone que, en estas familias, la transmisión es autosómica recesiva, no se puede descartar en forma absoluta la presencia de casos de transmisión dominante con penetrancia reducida. En $5-10 \%$ de estos pacientes con formas no-dominantes, se considera que se trata de una mutación "de novo". Un estudio realizado en Italia demostró que la ocurrencia de estas mutaciones no-dominantes es seis veces más frecuente que para las mutaciones recesivas. ${ }^{11}$

\section{ETIOPATOGENIA}

La membrana eritrocitaria mantiene la homeostasis celular a través de varios mecanismos, que incluyen retención de los componentes vitales, excreción de deshechos metabólicos, regulación del metabolismo y del $\mathrm{pH}$ eritrocitario, e importación del hierro para la síntesis de hemoglobina. ${ }^{6}$ El glóbulo rojo normal tiene forma de disco bicóncavo ("pelota desinflada"). En estas condiciones, la membrana no se encuentra tensionada, es altamente deformable y mantiene un estado de mínima energía, que proporciona una relación superficie/volumen máxima.

La membrana es una estructura compleja que comprende una doble capa lipídica, proteínas integrales y proteínas que constituyen el citoesqueleto (Tabla 1). Tanto las interacciones horizontales de las proteínas del citoesqueleto entre sí como las interacciones verticales entre ellas y las proteínas integrales de la membrana son esenciales para la integridad y estabilidad de la membrana y para la capacidad de deformación de la célula (Figura 1). ${ }^{6,12-14}$ Las uniones horizontales son paralelas al plano de la membrana y permiten que el eritrocito se extienda elásticamente para luego retornar a su forma inicial; por lo

TABla 1. Proteínas de la membrana eritrocitaria

\begin{tabular}{l|cc}
\hline Integrales & \multicolumn{2}{|c}{ Periféricas } \\
\hline $\begin{array}{l}\text { Total o parcialmente } \\
\text { sumergidas en la } \\
\text { bicapa lipídica }\end{array}$ & \multicolumn{2}{|c}{$\begin{array}{c}\text { Por fuera de la bicapa lipídica } \\
\text { en contacto en el citosol }\end{array}$} \\
\hline & \multicolumn{2}{|c}{} \\
Banda 3 & Citoesqueleto & Enzimas \\
Glicoforinas & Actina & Proteincinasas \\
Proteínas Rh & Ankirina & Hidrolasas \\
Acuaporina & Proteína 4.1 & Acetil- \\
& Proteína 4.2 & Gluceraldehido-3P \\
& Aducina & deshidrogenasa \\
& Tropomiosina & \\
& Tropomodulina & \\
& Otras & \\
\hline
\end{tabular}


tanto, soportan la integridad estructural de la célula y su exposición al estrés mecánico. Las uniones verticales son perpendiculares al plano de la membrana, estabilizan la bicapa lipídica y constituyen la unión o anclaje del citoesqueleto con la membrana citoplasmática.

Las dos principales funciones de la membrana son el mantenimiento de la integridad estructural y el control de la permeabilidad catiónica. ${ }^{12,15,16}$ Por lo tanto, cualquier alteración cuali- o cuantitativa de las proteínas de membrana se traduce en una menor capacidad de deformación del eritrocito y una relación superficie/volumen alterada. ${ }^{15}$ Así, la morfología eritrocitaria puede variar según se produzca ganancia o pérdida de superficie o de volumen (Figura 2). Las células que sufren una disminución de la relación superficie/ volumen (por ejemplo, esferocitos, estomatocitos) tienen muy limitada capacidad para atravesar los sinusoides esplénicos y, por lo tanto, son rápidamente destruidas. ${ }^{17}$ Por el contrario, el aumento en la relación superficie/volumen (por ejemplo, dianocitos) es generalmente inocuo y asintomático. Estas alteraciones en la estructura de la membrana pueden causar distintos tipos de anemias hemolíticas (Tabla 2), de las cuales la más frecuente es la ESH.

En la ESH, el defecto molecular primario genera un defecto cuali- o cuantitativo en las proteínas involucradas en mantener el citoesqueleto unido a la bicapa lipídica. ${ }^{14,18,19}$ Recientemente, se ha comunicado que las

FiguRA 2. Efectos de la alteración de los componentes de membrana

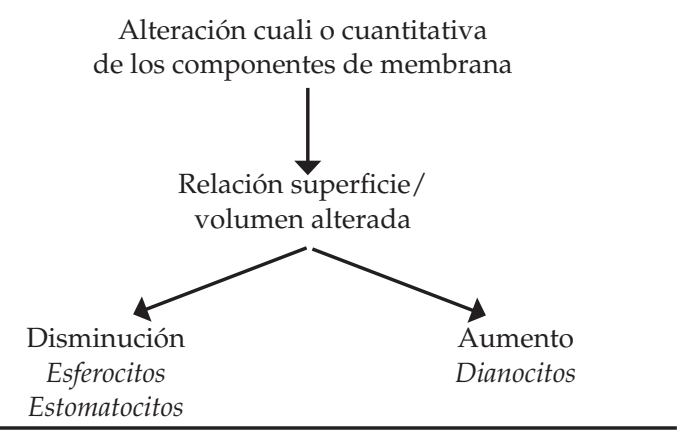

FIGURA 1. Estructura de la membrana del glóbulo rojo

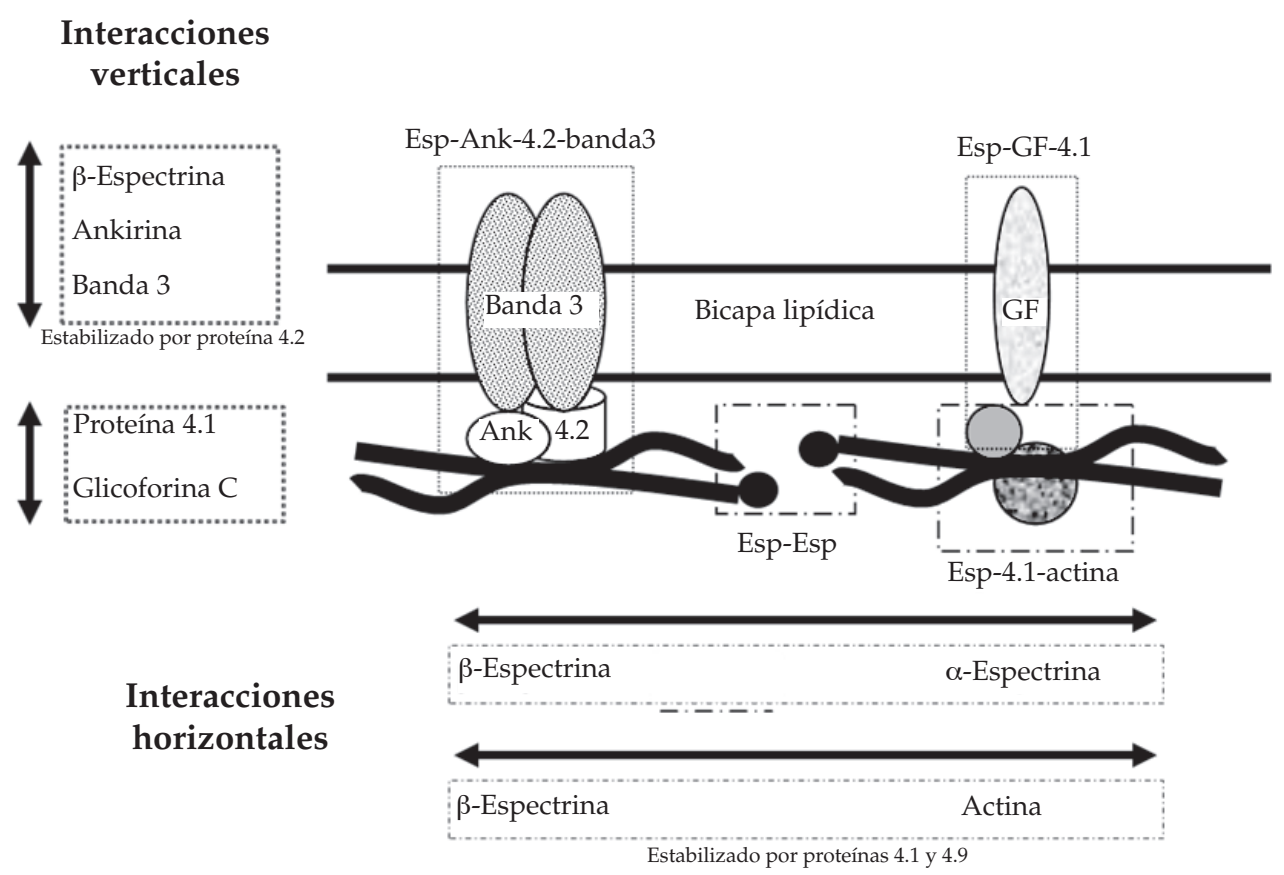

Esp: espectrina; Ank: ankirina; Gf: glicoforina. : interacciones verticales. : interacciones horizontales. 
deficiencias más frecuentes en nuestro país corresponden a ankirina y espectrina, ya sea en forma aislada o asociada a otras deficiencias (Figura 3).$^{8,9}$ Estos datos son coincidentes con la mayoría de los publicados en el mundo, que incluye países latinoamericanos,,$^{20-27}$ aunque difieren de otros en los que se ha comunicado que la deficiencia predominante es de banda $3 .{ }^{28-31}$

La deficiencia proteica produce, a su vez, alteraciones secundarias en la membrana. La teoría más aceptada dice que la ESH es causada por la desconexión entre el citoesqueleto y la capa bilipídica, seguida de vesiculación de los

TABla 2. Anemias hemoliticas hereditarias por trastornos de membrana eritrocitaria

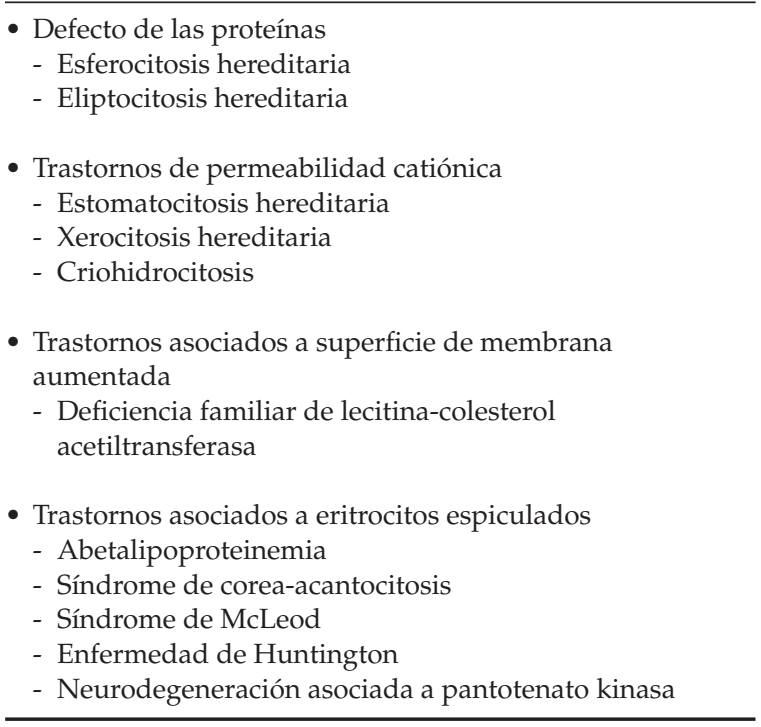

Figura 3. Prevalencia de deficiencias de proteínas de membrana detectadas en pacientes con esferocitosis hereditaria en Argentina
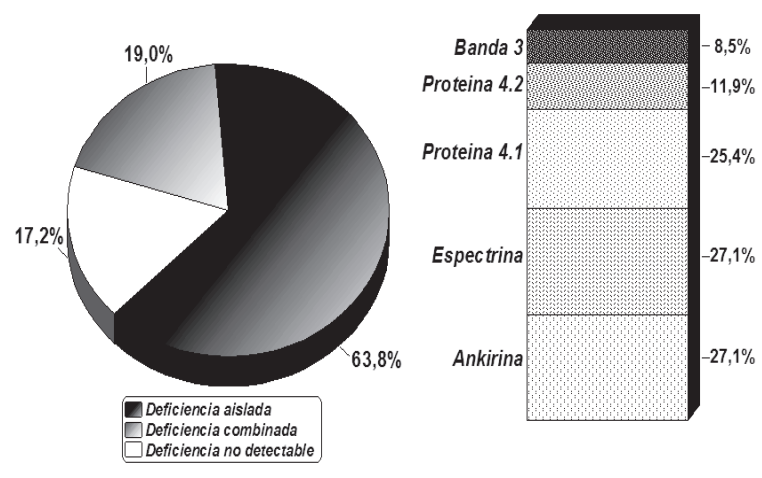

componentes de superficie que han perdido su soporte. ${ }^{6}$ Este fenómeno lleva a una progresiva reducción del área de superficie de membrana $\mathrm{y}$, como consecuencia, al cambio de forma del hematíe. La pérdida del 15-20\% de los lípidos de membrana, característica de la ESH, es simétrica, y no están alteradas las proporciones relativas de colesterol ni fosfolípidos. Otra de las consecuencias de estas alteraciones de la membrana es que se vuelve más permeable al sodio y al potasio que las células normales. Este excesivo flujo de $\mathrm{Na}^{+}$hacia el interior del eritrocito activa la ATPasa y, por ende, la bomba de $\mathrm{Na}^{+} / \mathrm{K}^{+}$. Por lo tanto, aumenta el consumo de ATP y, como consecuencia, se produce un aumento de la glicólisis para suplir la demanda de ATP. En el bazo, la mayor parte de la sangre arterial fluye directamente hacia los cordones esplénicos, una masa tortuosa de pasajes estrechos interconectados entre sí, formada por células reticulares y revestida por fagocitos. Para reingresar a la circulación, los eritrocitos deben atravesar espacios entre las células endoteliales que forman las paredes de los sinusoides venosos. ${ }^{32}$ Estos espacios, aun en su máximo punto de distensión, son invariablemente más pequeños que los glóbulos rojos, que deben, entonces, deformarse en gran medida para atravesarlos. ${ }^{33}$

Los esferocitos, al haber perdido su capacidad de deformarse, son selectivamente secuestrados en estos espacios. El tiempo de tránsito a través de los cordones esplénicos, que es de 30-40 segundos para un eritrocito normal, se encuentra prolongado a 15-150 minutos en el caso de los esferocitos. ${ }^{34}$ En consecuencia, el bazo de pacientes con ESH presenta cordones congestionados de modo masivo, pero con sinusoides relativamente vacíos, lo cual contrasta con la situación observable en bazos normales. Durante su detención en el bazo, los esferocitos sufren distintos cambios, que los llevan finalmente a su destrucción (Figura 4). ${ }^{67,715,32-38}$

\section{DATOS DE LABORATORIO}

La mayoría de los niños presentan anemia, con niveles de hemoglobina generalmente de 9-12 g/dL. ${ }^{39-41}$ En las formas de moderadas a graves, los valores están por debajo de $9 \mathrm{~g} / \mathrm{dL}$. Otros pacientes presentan un estado hemolítico compensado, con valores de hemoglobina dentro de los límites normales. ${ }^{41-43}$ Los recuentos reticulocitarios están siempre elevados y su nivel está en relación con la gravedad de la hemólisis. 
La excepción a esta situación se puede dar en los neonatos y durante las crisis aplásicas. La observación del extendido de sangre periférica suele ser fundamental para el diagnóstico. El hallazgo característico es la visualización de esferocitos, que son hematíes que han perdido su característica forma bicóncava y se muestran redondeados, hiperdensos, hipercrómicos, sin el halo claro central, a veces con contornos irregulares, y de tamaño inferior al eritrocito normal (Figura 5). Cabe destacar que, en los extendidos de pacientes con ESH, los esferocitos son prácticamente la única forma anormal de eritrocitos presente.

De los índices hematimétricos, solo la concentración de hemoglobina corpuscular media ( $\mathrm{CHCM}$ ) es patológica, con valores frecuentemente por encima de lo normal, que reflejan el grado relativo de deshidratación celular que presentan los hematíes. ${ }^{44-49}$ El volumen corpuscular medio (VCM) y la hemoglobina corpuscular media (HCM) están dentro de los valores normales. La amplitud de distribución eritrocitaria (ADE) está significativamente aumentada. ${ }^{47,49}$
En la ESH, la hemólisis es fundamentalmente extravascular, ya que es en el bazo donde habitualmente se produce la destrucción eritrocitaria. Por lo tanto, las pruebas indicativas de hemólisis que presentan estos pacientes son niveles elevados de bilirrubina indirecta y de urobilinógeno fecal.

FiguRA 5. Extendido de sangre periférica en un paciente con esferocitosis hereditaria. Se observa gran cantidad de esferocitos con su morfología característica $(\longrightarrow)$ y algunos con bordes irregulares $(\longrightarrow)$

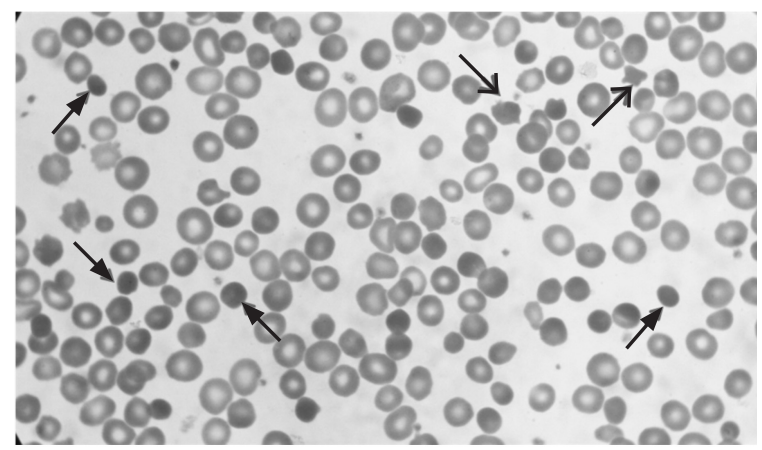

FIgURA 4. Fisiopatogenia de la destrucción eritrocitaria. El defecto proteico conduce a la formación de esferocitos. La disminución de la deformabilidad es responsable de un mayor tiempo de retención de la célula en el bazo. La eritrostasia conduce al secuestro esplénico (hemólisis extravascular) o bien a la liberación del eritrocito con una mayor pérdida de membrana

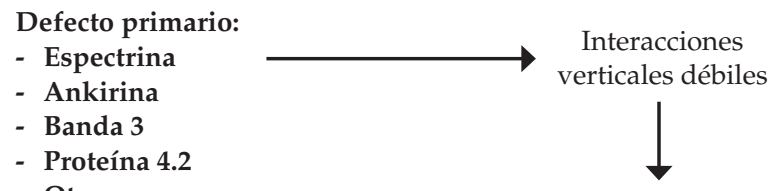

- Otras

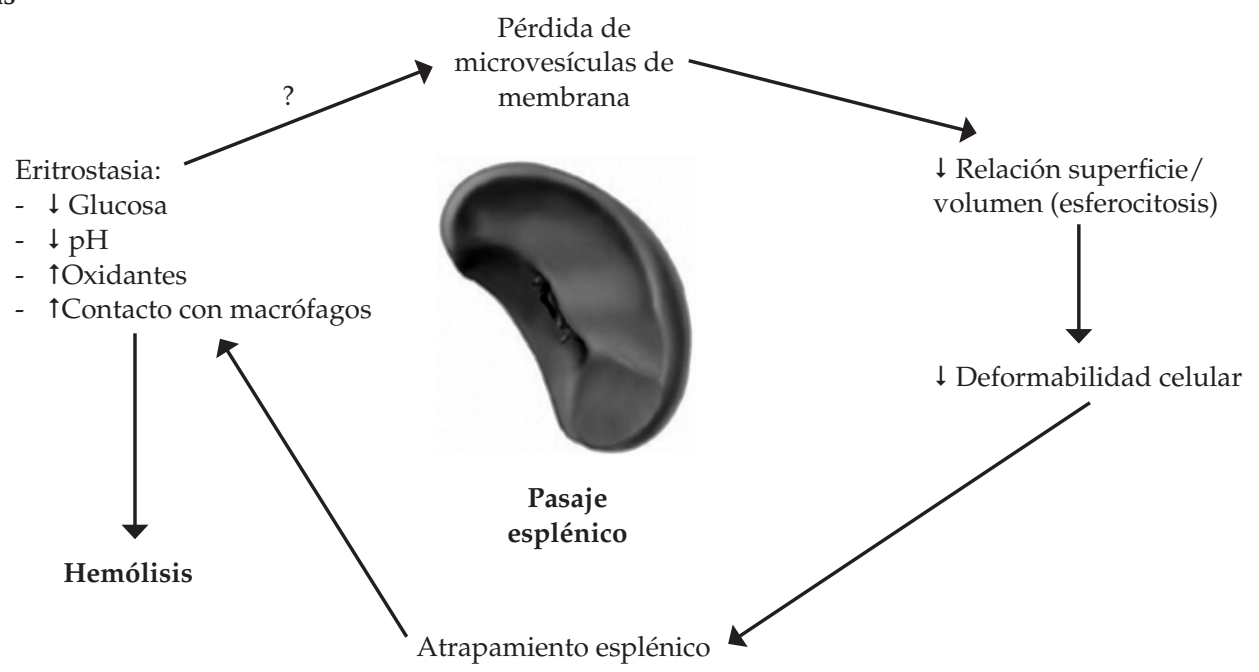


La ESH típica no presenta mayores problemas para el diagnóstico. En la mayoría de los casos, la asociación de historia familiar positiva, hallazgos clínicos característicos, evidencia de laboratorio de hemólisis extravascular, visualización de esferocitos en el extendido de sangre periférica y prueba de Coombs directa (PCD) negativa es suficiente para confirmar la enfermedad. ${ }^{13,50-52}$ Pero, en otros casos, el diagnóstico puede ser difícil. En la ESH leve, los pacientes pueden tener niveles de hemoglobina y bilirrubina normales y observarse muy pocos o ningún esferocito en el extendido (solo se visualizan en $25-30 \%$ de estos pacientes). $5,44,46,50$ Por el contrario, en la ESH grave, se pueden observar, además de los esferocitos, eritrocitos con otras alteraciones morfológicas (acantocitos, equinocitos, poiquilocitos, etc.), y se plantea, entonces, el diagnóstico diferencial con otras anemias hemolíticas. . $^{12,50,53,54}$ En estas situaciones, es imprescindible realizar las pruebas especiales confirmatorias del diagnóstico (Tabla 3). ${ }^{41}$

Sin embargo, ninguna de estas pruebas puede detectar el 100\% de los pacientes. ${ }^{6,13,50,55-57} \mathrm{La}$ fragilidad osmótica eritrocitaria (FOE), que da resultados positivos en aproximadamente $80 \%$ de los pacientes, ${ }^{8,15,58}$ es aún considerada el "patrón oro" por algunos autores, ${ }^{50,55,59}$ realizada simultáneamente con la autohemólisis (AH), aunque no sirve para diferenciar ESH de la esferocitosis secundaria a otras patologías, como anemia hemolítica autoinmune (AHAI), enfermedad hemolítica del recién nacido por incompatibilidad ABO (EHRN-ABO) o eliptocitosis hereditaria esferocítica. ${ }^{59,60}$ Actualmente, se dispone de nuevas pruebas diagnósticas, más específicas y sensibles, que presentan, además, la ventaja de requerir menor cantidad de sangre para el procesamiento. ${ }^{8,61-65}$

TABla 3. Pruebas de laboratorio especializado utilizadas para confirmar el diagnóstico de esferocitosis hereditaria

- Pruebas de fragilidad osmótica eritrocitaria (basal y diferida)

- Autohemólisis a 48 h con corrección por glucosa

- Criohemólisis hipertónica

- Lisis con glicerol acidificado

- Ectacitometría

- Citometría de flujo con 5'eosina maleimida

- Fragilidad osmótica por citometría de flujo

- Electroforesis de proteínas de membrana en gel de poliacrilamida

- Determinación del defecto molecular
En nuestro país, algunas de estas -criohemólisis hipertónica $(\mathrm{CH})$, citometría de flujo con 5'eosina maleimida (5'EMA-CF), FOE por citometría de flujo (FOE-CF)- se comenzaron a implementar a partir del año 2007.8,9,65-67 A continuación, se describen varias de las pruebas disponibles para poder interpretar correctamente sus resultados.

Prueba de fragilidad osmótica eritrocitaria: En el eritrocito normal, las características de su membrana le brindan una abundante superficie en relación con su volumen, mientras que, en el esferocito, esta relación superficie/volumen está disminuida. Cuando un eritrocito normal se suspende en una solución isotónica, conserva su forma y tamaño y no ocurre destrucción, ya que el balance de entrada y salida de agua es nulo. Si la solución es hipotónica, el agua penetra en el eritrocito para diluir su concentración de sales y equilibrar su presión osmótica con la del medio, lo que lleva a un aumento progresivo de volumen del hematíe hasta que su capacidad se agota, adopta la forma esférica y se rompe y libera hemoglobina al medio. La prueba de FOE se realiza suspendiendo los hematíes en soluciones de ClNa gradualmente más hipotónicas. Las células que ya tienen una relación superficie/volumen disminuida, como los esferocitos o estomatocitos, alcanzan la forma esférica límite con menor ingreso de agua en concentraciones de $\mathrm{ClNa}$ mayores que las células normales, y se presenta, entonces, lo que se denomina una fragilidad osmótica aumentada. Por el contrario, cuando la relación superficie/volumen está aumentada (por ejemplo, dianocitos), los eritrocitos son más resistentes que los normales y solo se lisan en soluciones muy hipotónicas y presentan así una fragilidad osmótica disminuida. En el corto tiempo de incubación de la prueba, no se produce intercambio de cationes y solo hay movimiento de agua; por lo tanto, la hiperactividad de la bomba de sodio de los esferocitos no interviene en el mecanismo de hemólisis y no influye en el resultado de la determinación. Si la prueba se realiza con sangre recién extraída (prueba de FOE inmediata), será positiva solo en un $75 \%$ de pacientes con ESH. ${ }^{43,68}$ Para evitar este elevado porcentaje de falsos negativos, se debe siempre realizar la prueba de FOE diferida. Al dejar la sangre incubando 24 horas a $37^{\circ} \mathrm{C}$, se genera déficit de glucosa, y las condiciones de estrés metabólico conducen a la pérdida de membrana de la población esferocítica, lo que aumenta su fragilidad. Como este proceso tiene muy 
escaso efecto sobre los eritrocitos normales, así se enfatiza la diferencia entre ambas poblaciones. Por este método, también se pone en evidencia la población esferocítica de los pacientes con recuentos reticulocitarios elevados, cuya curva de FOE no incubada puede ser normal debido a que los reticulocitos presentan una fragilidad osmótica inferior a la normal y pueden, entonces, compensar los resultados. Los parámetros para tener en cuenta para evaluar los resultados de una prueba de FOE son los siguientes: ${ }^{69}$

- Desplazamiento: La curva está desplazada hacia la derecha (concentraciones mayores de $\mathrm{ClNa}$ ) respecto a la normal cuando la FOE está aumentada (esferocitos) o hacia la izquierda cuando está disminuida (talasemias, ferropenia). En la Figura 6, se muestran distintos tipos de curvas típicas.
- Fragilidad corpuscular media: Es la concentración de $\mathrm{ClNa}$ a la cual se produce un $50 \%$ de hemólisis. El valor obtenido es más elevado cuanto más osmóticamente frágil es la población eritrocitaria. Las patologías con eritrocitos osmóticamente resistentes (por ejemplo, talasemias) presentan valores disminuidos.

- Hemólisis incipiente: Es la concentración de $\mathrm{ClNa}$ a la cual visualmente se detecta que comienza la hemólisis; es mayor cuanto más osmóticamente frágil es la población eritrocitaria.

Prueba de autohemólisis: $\mathrm{Si}$ se dejan eritrocitos normales incubando a $37^{\circ} \mathrm{C}$ durante 48 horas, se produce cierto grado de hemólisis espontánea, no mayor de $2,5 \%$. Los esferocitos presentan hiperactividad de la bomba de $\mathrm{Na}^{+}$y una glucólisis
Tipo I

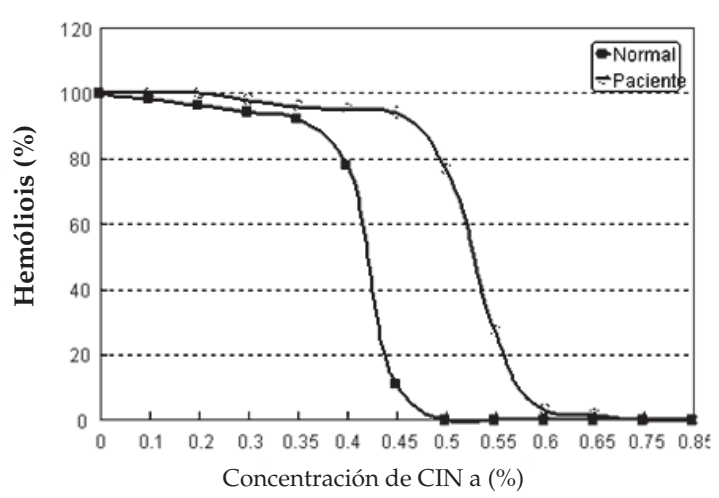

Tipo III

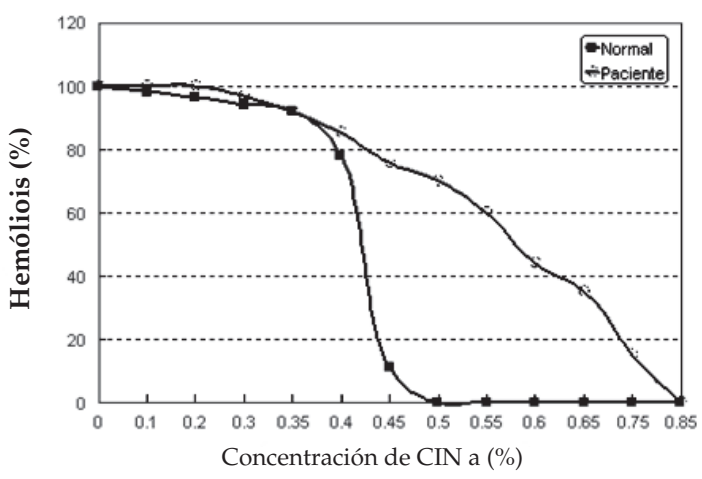

Tipo II

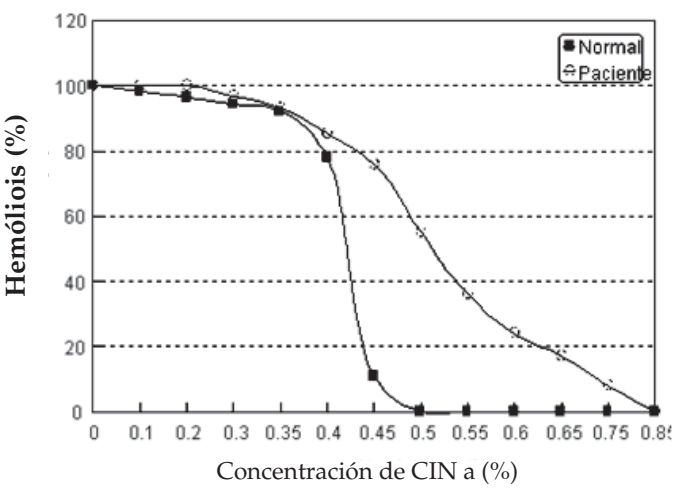

Talasemia

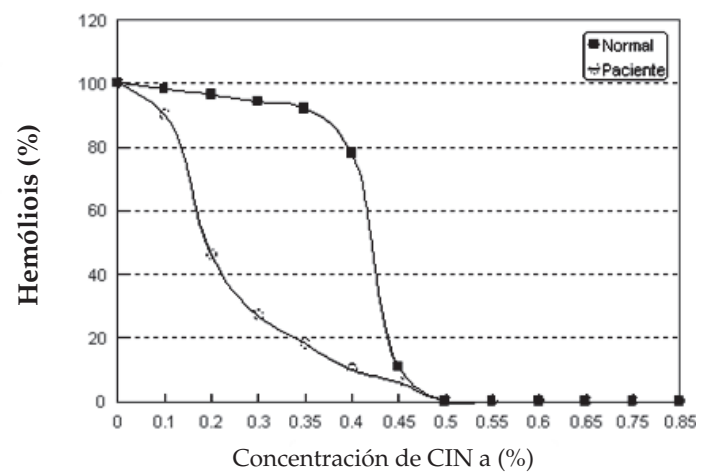

TIPO I: Curva con la forma sigmoidea normal pero desplazada a la derecha. En el extendido de sangre periférica, se observa un pequeño porcentaje de esferocitos. TIPO II: Se mantiene cierto grado de hemólisis en concentraciones $\geq 60 \%$ (cercanas a la isotónica), lo que genera una prolongación o cola de la curva. Esto se debe a la presencia de esferocitos que tienen condicionamiento esplénico y se encuentran en estado prelítico. En el extendido, se observa heterogeneidad eritrocitaria. TIPO III: Curva con pérdida de la forma sigmoidea. Se observa en las esferocitosis graves. En el extendido, se visualiza un alto porcentaje de esferocitos. TALASEMIAS: Se observa una curva desplazada hacia la izquierda debido a que la población eritrocitaria posee una mayor resistencia osmótica. 
aumentada, ya que requiere energía (ATP) para balancear el flujo de $\mathrm{Na}^{+}$. En consecuencia, durante la incubación, el eritrocito sufre estrés metabólico y, cuando toda la glucosa del medio se ha consumido, no puede equilibrar el movimiento de cationes y se produce la lisis osmótica. La deprivación de glucosa en el medio es más rápida que para el control normal, ya que el consumo de glucosa basal del esferocito es mayor.

De esta manera, la lisis del esferocito se inicia antes y progresa más rápidamente que la del eritrocito normal, con lo cual la autohemólisis se incrementa hasta valores de $45 \% .43,70 \mathrm{El}$ agregado de glucosa a esta solución de hematíes corrige el grado de autohemólisis en la ESH y algunas otras raras patologías de membrana eritrocitaria, pero no en AHAI y en la mayoría de las otras anemias hemolíticas hereditarias. ${ }^{43,70}$ En resumen, una $\mathrm{AH}$ aumentada que corrige con glucosa es altamente sugestiva de ESH, mientras que la no corrección orienta el diagnóstico hacia otras enfermedades de membrana. Sin embargo, en algunos pacientes con gran cantidad de esferocitos en estado prelítico, puede no observarse corrección con glucosa; en estos casos, la población esferocítica se evidencia en la curva basal de FOE.

Prueba del glicerol acidificado: Es otro método para confirmar la fragilidad osmótica aumentada. ${ }^{71}$ El agregado de glicerol a una suspensión de eritrocitos en medio hipotónico, al retardar la entrada de agua a la célula, prolonga el tiempo de lisis celular y permite medirlo con exactitud. El valor normal de referencia es mayor de 30 minutos; para el diagnóstico de ESH, se requiere un tiempo inferior a cinco minutos. $\mathrm{Su}$ sensibilidad y especificidad son similares a las de la FOE, pero aún existen controversias relativas a su utilidad. $56,72,73$

Criohemólisis hipertónica: Evalúa la hemólisis de los eritrocitos suspendidos en un medio hipertónico, a los que se somete a un brusco cambio de temperatura. En estas condiciones, los esferocitos son más susceptibles a la hemólisis que los eritrocitos normales. La prueba no depende de la relación superficie/volumen, sino de la integridad de las proteínas de membrana, que repercute notablemente en su plasticidad (Figura 7). Esta prueba presenta la ventaja de arrojar resultados normales cuando la presencia de esferocitos se relaciona con AHAI.

Citometría de flujo con 5'eosina maleimida: Esta prueba evalúa la intensidad de fluorescencia emitida por el reactivo 5'EMA, que interacciona covalentemente con las proteínas banda
3 (principalmente), RhAG, Rh y CD47, de la membrana eritrocitaria. ${ }^{60}$ En la ESH, cualquiera sea la proteína deficiente primaria, se obtiene una disminución de la fluorescencia debido a la pérdida de membrana que contiene las proteínas que reaccionan con el reactivo fluorescente. Es importante destacar que, en otras anemias hemolíticas, la fluorescencia emitida es normal, de manera que permite diferenciar los esferocitos de la ESH de aquellos presentes en la AHAI. Los resultados se informan como porcentaje de disminución de la intensidad de fluorescencia respecto del promedio obtenido para seis controles normales procesados de modo simultáneo y que posean el mismo tiempo de almacenamiento que la muestra por testear. ${ }^{60} \mathrm{La}$ prueba es indudablemente positiva para ESH si presenta una disminución de fluorescencia superior al 21\%; sin embargo, el valor de corte a partir del cual la prueba es considerada negativa es variable, por lo que cada laboratorio debe establecer su propio valor. $8,56,60,74$

Fragilidad osmótica eritrocitaria por citometría de flujo: Esta prueba cuantifica los eritrocitos resistentes a la hemólisis que se genera por el agregado de agua a una suspensión de sangre en solución fisiológica. Es decir que se evalúa el porcentaje de eritrocitos remanentes, en lugar de evaluar la hemólisis producida en el medio hipotónico. La prueba fue descrita hace muy pocos años, ${ }^{64}$ de manera que aún no se ha difundido ampliamente. Los resultados han sido reproducidos por diferentes grupos. ${ }^{64,67,75,76}$ En el único estudio realizado en nuestro país, la prueba mostró una sensibilidad de $89 \%$ y una especificidad de $85 \%$ para el diagnóstico. ${ }^{9} \mathrm{Su}$ mayor ventaja reside en que permite evaluar la fragilidad osmótica con una mínima cantidad de sangre, lo que constituye una herramienta diagnóstica muy útil para ser utilizada, en especial, en niños pequeños, ya que requiere un volumen de muestra considerablemente menor que el necesario para las curvas de FOE. ${ }^{66,67}$

Ectacitometría: En el ectacitómetro, se someten las células a una fuerza lateral constante por rotación entre dos cilindros concéntricos que las contienen, mientras que la osmolaridad del medio en que están suspendidas se incrementa gradualmente. La deformabilidad de los eritrocitos está gobernada por tres factores: la relación superficie/volumen, la viscosidad citoplasmática y la rigidez intrínseca de la membrana. Con este procedimiento, se obtiene un perfil de deformabilidad osmótica, que presenta 
tres parámetros para evaluar (Figura 8.A): $:^{77}$

- DImáx (índice de deformabilidad máximo): Se alcanza en el medio isotónico y depende del área de membrana disponible del eritrocito.

- Omin (osmolaridad mínima): Es la osmolaridad a la cual el DI es mínimo. Se produce en el medio hipotónico cuando el eritrocito alcanza su volumen crítico. Depende de la relación superficie/volumen y coincide con la fragilidad corpuscular media (FCM) de las curvas FOE.

- O': Osmolaridad a la cual la DI es igual a la mitad de la DImáx. Este parámetro depende de la viscosidad intracelular y, por lo tanto, se relaciona con la CHCM.

En la ESH, el perfil obtenido mostrará un menor DImáx acorde a la pérdida de membrana, una Omin mayor debido a la fragilidad osmótica aumentada y una $\mathrm{O}^{\prime}$ menor, que refleja la presencia de eritrocitos con mayor CHCM (Figura 8.B).

Electroforesis en gel de poliacrilamida de membranas eritrocitarias: Consiste en fraccionar las proteínas de la membrana eritrocitaria por electroforesis en condiciones desnaturalizantes y cuantificarlas por densitometría, lo que permite certificar la disminución de una o más de las proteínas. Es una técnica muy laboriosa que arroja resultados positivos en poco más del $70 \%$ de los pacientes. ${ }^{8,20,21,29,59,78-82}$ Por lo tanto, su realización queda restringida a aquellos casos con fuerte presunción diagnóstica que no han podido ser confirmados mediante las pruebas habituales. ${ }^{13}$ Estudios recientes sugieren que la cuantificación relativa de las proteínas podría ser de utilidad como valor predictivo de la evolución de la enfermedad. ${ }^{30}$

Diagnóstico molecular: En la ESH, se han descrito múltiples alteraciones moleculares. ${ }^{12,13,83}$ La diversidad de los genes involucrados y la gran cantidad de mutaciones reportadas para cada gen limitan la posibilidad de utilizar esta metodología para el diagnóstico. Además, son defectos generalmente restringidos a una familia específica y no predicen gravedad, por lo que no tienen mayor importancia clínica, excepto la de

\section{Control normal}
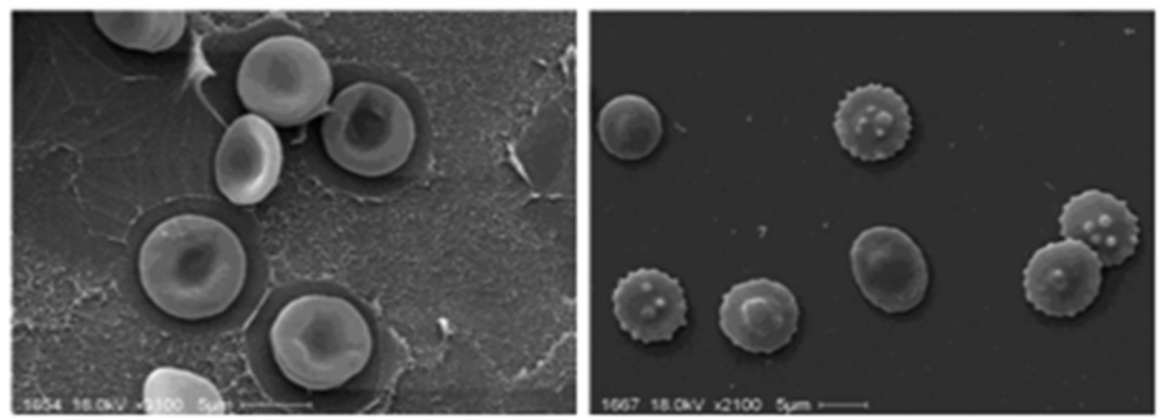

Esferocitosis hereditaria
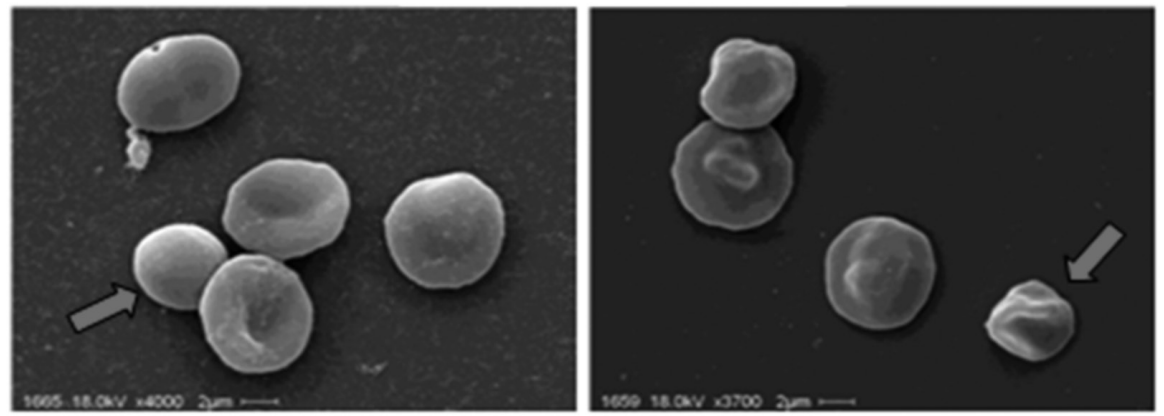

Se observan los eritrocitos en su propio plasma de un control normal y de un paciente con esferocitosis hereditaria antes y después de ser sometidos a deshidratación y enfriamiento en una solución hipertónica. Obsérvese la apariencia rígida de los esferocitos, que contrasta con la deformación "plástica" de los eritrocitos normales. 

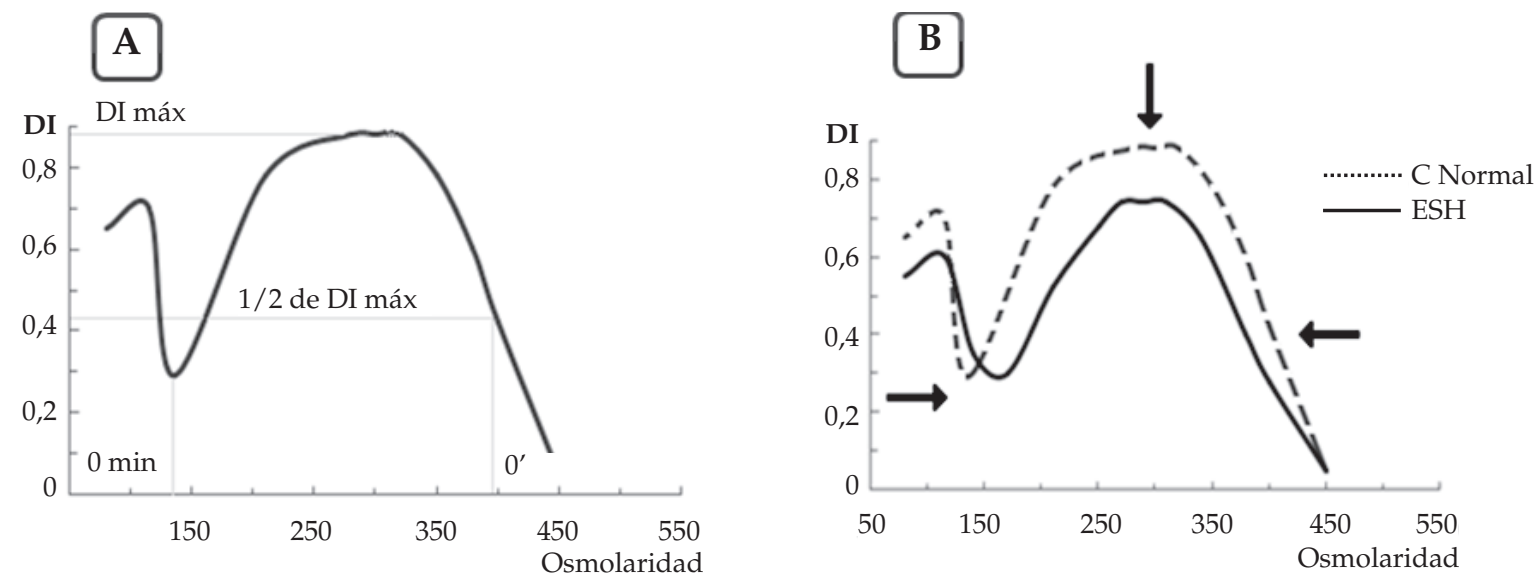

A: parámetros para evaluar; B: curvas que comparan control normal con un paciente con esferocitosis hereditaria.

DI: índice de deformabilidad.

ESH: esferocitosis hereditaria.

brindar un asesorami ento genético más preciso a la familia afectada. ${ }^{50}$ Estos estudios se efectúan en el ámbito de la investigación clínica y solo son de utilidad en los casos inusuales de mutaciones "de novo" o en los heterocigotas compuestos. ${ }^{13}$

La sensibilidad y especificidad de las pruebas arriba descritas varían según los distintos autores. Como ninguna de ellas es positiva en $100 \%$ de los casos, ${ }^{31,49-51,55,62}$ en la práctica clínica, cada laboratorio estudia al paciente con una pequeña batería de pruebas, de acuerdo con la sensibilidad y especificidad propias alcanzadas con cada una de ellas y con la disponibilidad de equipos y reactivos. Las pruebas más recomendadas actualmente son la 5'EMA-CF y la $\mathrm{CH}$, ya que han demostrado alta sensibilidad y especificidad. ${ }^{13,84}$ En un estudio realizado en Argentina, la asociación de ambas pruebas permitió confirmar el diagnóstico en 93,5\% de los pacientes con ESH. ${ }^{8}$ Actualmente, en algunos laboratorios de nuestro país, las pruebas de rutina que se pueden realizar son $\mathrm{FOE}, \mathrm{AH}$, 5'EMA-CF, CH y FOE-CF. Además, para el estudio de recién nacidos o niños pequeños, en quienes la toma de muestras de sangre puede ser dificultosa, se ha demostrado la utilidad del uso de sangre capilar para la realización de algunas de estas pruebas. ${ }^{65-67} \mathrm{El}$ uso de un muy pequeño volumen de sangre capilar $(300 \mathrm{uL})$ para realizar simultáneamente 5'EMA-CF, $\mathrm{CH}$ y FOE-CF permite confirmar el diagnóstico en el
$100 \%$ de los pacientes estudiados. ${ }^{65-67}$ Por lo tanto, recomendamos, como rutina actual en estos casos, la realización simultánea de estas tres pruebas utilizando sangre capilar.

\section{REFERENCIAS}

1. Petz LD, Garratty G. Historical concepts of immune hemolytic anemias. En: Petz LD, Garratty G, eds. Immune Hemolytic Anemias. $2^{\text {nd }}$ ed. Philadelphia: Churchill Livingstone; 2004. Págs.1-31.

2. Gallagher PG. Abnormalities of the erythrocyte membrane. Pediatr Clin North Am 2013;60(6):1349-62.

3. Morton NE, MacKinney AA, Kosower N, Schilling RF, et al. Genetics of spherocytosis. Am J Hum Genet 1962;14:170-84.

4. Godal HC, Heisto H. High prevalence of increased osmotic fragility of red blood cells among Norwegian donors. Scand J Haematol 1981;27(4):30-4.

5. Eber SW, Pekrun A, Neufeldt A, Schröter W. Prevalence of increased osmotic fragility of erythrocytes in German blood donors: screening using a modified glycerol lysis test. Ann Hematol 1992;64(2):88-92.

6. Gallagher PG. Disorders of erythrocyte metabolism and shape. En: Christensen RD, ed. Hematologic Problems of the Neonate. Philadelphia: WB Saunders; 2000. Págs.209-38.

7. Glader B, Allen G. Neonatal hemolysis. En: de Alarcón PA, Werner EJ, eds. Neonatal Hematology. Cambridge: Cambridge University Press; 2005. Págs.132-62.

8. Crisp RL, Solari L, Vota D, García E, et al. A prospective study to assess the predictive value for hereditary spherocytosis using five laboratory tests (cryohemolysis test, eosin-5'-maleimide flow cytometry, osmotic fragility test, autohemolysis test, and SDS-PAGE) on 50 hereditary spherocytosis families in Argentina. Ann Hematol 2011;90(6):625-34.

9. Donato H, Crisp RL, Garcia E, Rapetti MC, et al. Aspectos demográficos, clínicos, diagnósticos y terapéuticos de la 
esferocitosis hereditaria en nuestro país. Estudio sobre 143 casos. Hematologia (B. Aires) 2014;18(1):9-16.

10. Stevens RF, Evans DI. Congenital spherocytosis is often not hereditary. Clin Pediatr 1981;20(1):47-9.

11. Miraglia del Giudice E, Nobili B, Francese M, D'Urso L, et al. Clinical and molecular evaluation of non-dominant hereditary spherocytosis. Br J Haematol 2001;112(1):42-7.

12. Gallagher PG, Forget BG, Lux SE. Disorders of the erythrocyte membrane. En: Nathan DG, Orkin SH, eds. Nathan and Oski's Hematology of Infancy and Childhood. $5^{\text {th }}$ ed. Philadelphia: WB Saunders; 1998. Págs.544-664.

13. Bolton-Maggs PH, Stevens RF, Dodd NJ, Lamont G, et al. Guidelines for the diagnosis and management of hereditary spherocytosis. Br J Haematol 2004;126(4):455-74.

14. Liu SC, Derick LH, Agre P, Palek J. Alteration of the erythrocyte membrane skeletal ultrastructure in hereditary spherocytosis, hereditary elliptocytosis, and pyropoikilocytosis. Blood 1990;76(1):198-205.

15. Lux SE, Palek J. Disorders of the red cell membrane. En: Handin RI, Lux SE, Stossel TP, eds. Blood: Principles and Practice of Hematology. Philadelphia: JB Lippincott; 1995. Págs.1701-818.

16. Gallagher PG, Ferreira JD. Molecular basis of erythrocyte membrane disorders. Curr Opin Hematol 1997;4(2):128-35.

17. Payne LG, Hayward CP, Kelton JG. Destruction of red cells by the vasculature and reticuloendothelial system. En: Nathan DG, Orkin SH, eds. Nathan and Oski's Hematology of Infancy and Childhood. 5th ed. Philadelphia: WB Saunders; 1998. Págs.523-43.

18. Delaunay J, Alloisio N, Morle L, Baklouti F, et al. Molecular genetics of hereditary elliptocytosis and hereditary spherocytosis. Ann Genet 1996;39(4):209-21.

19. Tse WT, Lux SE. Red blood cell membrane disorders. Br J Haematol 1999;104(1):2-13.

20. Costa FF, AgreP, Watkins PC, WinkelmannJC, etal. Linkage of dominant hereditary spherocytosis to the gene for the erythrocyte membrane-skeleton protein ankyrin. $\mathrm{N} \mathrm{Engl} \mathrm{J}$ Med 1990;323(15):1046-50.

21. Miraglia del Giudice F, Iolascon A, Pinto L, Nobili B, et al. Erythrocyte membrane protein alterations underlying clinical heterogeneity in hereditary spherocytosis. $\mathrm{Br} J$ Haematol 1994;88(1):52-5.

22. Saad ST, Costa FF, Vicentim DL, Salles TS, et al. Red cell membrane protein abnormalities in hereditary spherocytosis in Brazil. Br J Haematol 1994;88(2):295-9.

23. Lanciotti M, Perutelli P, Valetto A, Di Martino D, et al. Ankyrin deficiency is the most common defect in dominant and nondominant hereditary spherocytosis. Haematologica 1997;82(4):460-2.

24. Dhermy D, Burnier O, Bougeois M, Grandchamp B. The red blood cell band 3 variant (band 3Biceêtrel: R490C) associated with dominant hereditary spherocytosis causes defective membrane targeting of the molecule and a dominant negative effect. Mol Membr Biol 1999;16(4):305-12.

25. Premetis E, Stamoulakatou A, Loukopoulos D. Erythropoiesis: hereditary spherocytosis in Greece: collective data on a large number of patients. Hematology 1999;4(4):361-6.

26. Ricard MP, Gilsanz F, Millan I. Erythroid membrane protein defects in hereditary spherocytosis. A study of 62 Spanish cases. Haematologica 2000;85(9):994-5.

27. Sánchez-López JY, Camacho AL, Magaña MT, Ibarra B, et al. Red cell membrane protein deficiencies in Mexican patients with hereditary spherocytosis. Blood Cells Mol Dis 2003;31(3):357-9.

28. CynoberT,Mohandas N, Tchernia G. Red cell abnormalities in hereditary spherocytosis: relevance to diagnosis and understanding of the variable expression of clinical severity.
J Lab Clin Med 1996;128(3):259-69.

29. Yawata Y, Kanzaki A, Yawata A, Doerfler W, et al. Characteristic features of the genotype and phenotype of hereditary spherocytosis in the Japanese population. Int $J$ Hematol 2000;7:118-35.

30. Rocha S, Rebelo I, Costa E, Catarino C, et al. Protein deficiency balance as a predictor of clinical outcome in hereditary spherocytosis. Eur J Haematol 2005;74(5):374-80.

31. Mariani M, Barcellini W, Vercellati C, Marcello AP, et al. Clinical and hematologic features of 300 patients affected by hereditary spherocytosis grouped according to the type of the membrane protein defect. Haematologica 2008;93(9):1310-7.

32. Chen LT, Weiss L. The role of the sinus wall in the passage of erythrocytes through the spleen. Blood 1973;41(4):529-37.

33. Weiss L, Tavassoli M. Anatomical hazards to the passage of erythrocytes through the spleen. Semin Hematol 1970;7(4):372-80.

34. Ferrant A, Leners N, Michaux JL, Verwilghen RL, et al. The spleen and haemolysis: evaluation of the intrasplenic transit time. Br J Haematol 1987;65(1):31-4.

35. Mayman D, Zipursky A. Hereditary spherocytosis: the metabolism of erythrocytes in the peripheral blood and in the splenic pulp. Br J Haematol 1974;27(2):201-17.

36. Lusher JM, Barnhart MI. The role of the spleen in the pathophysiology of hereditary spherocytosis and hereditary elliptocytosis. Am J Pediatr Hematol Oncol 1980;2:31-9.

37. Myhre Jensen O, Kristensen J. Red pulp of the spleen in autoimmune haemolytic anaemia and hereditary spherocytosis: morphometric light and electron microscopy studies. Scand J Haematol 1986;36(3):263-6.

38. Palek J, Jarolim P. Clinical expression and laboratory detection of red blood cell membrane protein mutations. Semin Hematol 1993;30(4):249-83.

39. Packman $\mathrm{CH}$. The spherocytic haemolytic anaemias. $\mathrm{Br} \mathrm{J}$ Haematol 2001;112(4):888-99.

40. Donato H, Crisp R, Rapetti MC. Anemias hemolíticas hereditarias por trastornos de membrana. En: Donato H, Rapetti MC, Crisp RL, eds. Anemias en Pediatría. Buenos Aires: Journal; 2013.Págs.105-34.

41. Da Costa L, Galimand J, Fenneteau O, Mohandas N. Hereditary spherocytosis, elliptocytosis, and other red cell membrane disorders. Blood Rev 2013;27(4):167-78.

42. Krueger HC, Burgert EO Jr. Hereditary spherocytosis in 100 children. Mayo Clin Proc 1966;41(12):821-30.

43. Young LE, Izzo MJ, Platzer RF. Hereditary spherocytosis. I. Clinical, hematologic and genetic features in 28 cases, with particular reference to the osmotic and mechanical fragility of incubated erythrocytes. Blood 1951;6(11):1073-98.

44. MacKinney AA Jr, Morton NE, Kosower NS, Schilling RF. Ascertaining genetic carriers of hereditary spherocytosis by statistical analysis of multiple laboratory tests. J Clin Invest 1962;41:554-67.

45. Diamond LK. Indications for splenectomy in childhood: Results in fifty-two operated cases. Am J Surg 1938;39:400-21.

46. Mohandas N, Kim YR, Tycko DH, Orlik J, et al. Accurate and independent measurement of volume and hemoglobin concentration of individual red cells by laser light scattering. Blood 1986;68(2):506-13.

47. Gilsanz F, Ricard MP, Millan I. Diagnosis of hereditary spherocytosis with dual-angle differential light scattering. Am J Clin Pathol 1993;100(2):119-22.

48. Pati AR, Patton WN, Harris RI. The use of the technicon $\mathrm{H} 1$ in the diagnosis of hereditary spherocytosis. Clin Lab Haematol 1989;11(1):27-30.

49. Michaels LA, Cohen AR, Zhao H, Raphael RI, et al. Screening for hereditary spherocytosis by use of automated 
erythrocyte indexes. J Pediatr 1997;130(6):957-60.

50. Grace RF, Lux SE. Disorders of the red cell membrane. En: Orkin SH, Nathan DG, Ginsburg D, Look AT, et al, eds. Nathan and Oski's Hematology of Infancy and Childhood. $7^{\text {th }}$ ed. Philadelphia: WB Saunders; 2009.Págs.659-837.

51. Hassoun H, Palek J. Hereditary spherocytosis: a review of the clinical and molecular aspects of the disease. Blood Rev 1996;10(3):129-47.

52. Gallagher PG. Red cell membrane disorders. Hematology Am Soc Hematol Educ Program 2005;13-8.

53. Agre P, Orringer EP, Bennett V. Deficient red-cell spectrin in severe, recessively inherited spherocytosis. N Engl JMed 1982;306(19):1155-61.

54. Coetzer TL, Lawler J, LiuSC, PrchalJT, et al. Partial ankyrin and spectrin deficiency in severe, atypical hereditary spherocytosis. N Engl J Med 1988;318(4):230-4.

55. Iolascon A, Avvisati RA. Genotype/phenotype correlation in hereditary spherocytosis. Haematologica 2008;93(9):1283-8.

56. Bianchi P, Fermo E, Vercellati C, Marcello AP, et al. Diagnostic power of laboratory tests for hereditary spherocytosis: a comparison study in 150 patients grouped according to molecular and clinical characteristics. Haematologica 2012;97(4):516-23.

57. King MJ, Zanella A. Hereditary red cell membrane disorders and laboratory diagnostic testing. Int J Lab Hematol 2013;35(3):237-43.

58. Korones D, Pearson HA. Normal erythrocyte osmotic fragility in hereditary spherocytosis. J Pediatr 1989;114(2):264-6.

59. Perrotta S, Gallagher PG, Mohandas N. Hereditary spherocytosis. Lancet 2008;372(9647):1411-26.

60. King MJ, Behrens J, Rogers C, Flynn C, et al. Rapid flow cytometric test for the diagnosis of membrane cytoskeleton-associated haemolytic anaemia. Br J Haematol 2000;111(3):924-33.

61. Streichman S, Gescheidt Y. Cryohemolysis for the detection of hereditary spherocytosis: correlation studies with osmotic fragility and autohemolysis. Am J Hematol 1998;58(3):206-12.

62. King MJ, Telfer P, MacKinnon $H$, Langabeer L, et al. Using the eosin-5-maleimide binding test in the differential diagnosis of hereditary spherocytosis and hereditary pyropoikilocytosis. Cytometry B Clin Cytom 2008;74(4):244-50.

63. King MJ,SmytheJS, Mushens R. Eosin-5-maleimidebinding to band 3 and Rh-related proteins forms the basis of a screening test for hereditary spherocytosis. Br J Haematol 2004;124(1):106-13.

64. Won DI, Suh JS. Flow cytometric detection of erythrocyte osmotic fragility. Cytometry B Clin Cytom 2009;76(2):135-41.

65. Crisp RL, Solari L, Gammella D, Schvartzman GA, et al. Use of capillary blood to diagnose hereditary spherocytosis. Pediatr Blood Cancer 2012;59(7):1299-301.

66. Crisp R, Solari L, Gammela D, Schvartzman G, et al. Use of capillary blood to perform the eosin-5'-maleimide flow cytometry (EMA-FC) test: results are comparable to venous blood. Haematologica 2010;95(Suppl 2):700, abs. 1808.

67. Crisp R, Solari L, Gammela D, Schvartzman G, et al. Flow cytometric osmotic fragility (FC-OF) can replace the traditional osmotic fragility (OF) test? Preliminary results. Haematologica 2010;95(Suppl 2):703, abs. 1816.

68. Young LE. Observations on inheritance and heterogeneity of chronic spherocytosis. Trans Assoc Am Physicians 1955;68:141-8.

69. Rapetti C, Donato H, Crisp R, Martorelli D. Anemias hemolíticas. En: Donato H, Rapetti C, eds. Anemias en Pediatría. Buenos Aires: Fundasap; 2005. Págs.87-134.

70. Altman KI, Izzo MJ, Swisher SN, Young LE. Studies on spontaneous in vitro autohemolysis in hemolytic disorders. Blood 1956;11(11):977-97.

71. Zanella A, Izzo C, Rebulla P, Zanuso F, et al. Acidified glycerol lysis test: a screening test for spherocytosis. $\mathrm{Br} \mathrm{J}$ Haematol 1980;45(3):481-6.

72. Zanella A, Milani S, Fagnani G, Mariani M, et al. Diagnostic value of the glycerol lysis test. J Lab Clin Med 1983;102(5):743-50.

73. Hoffmann JJ, Swaak-Lammers N, Breed WP, Strengers JL. Diagnostic utility of the pre-incubated acidified glycerol lysis test in haemolytic and non-haemolytic anaemias. Eur J Haematol 1991;47(5):367-70.

74. Girodon F, Garçon L, Bergoin E, Largier M, et al. Usefulness of the eosin-5'-maleimide cytometric method as a first-line screening test for the diagnosis of hereditary spherocytosis: comparison with ektacytometry and protein electrophoresis. Br J Haematol 2008;140(4):468-70.

75. Warang P, Gupta M, Kedar P, Ghosh K, et al. Flow cytometric osmotic fragility - An effective screening approach for red cell membranopathies. Cytometry B Clin Cytom 2011;80(3):186-90.

76. Shim YJ, Won DI. Flow cytometric osmotic fragility testing does reflect the clinical severity of hereditary spherocytosis. Cytometry B Clin Cytom 2013 Nov 15. [Epub ahead of print].

77. Clark MR, Mohandas N, Shohet SB. Osmotic gradient ektacytometry: comprehensive characterization of red cell volume and surface maintenance. Blood 1983;61(5):899-910.

78. Hanspal M, YoonSH, YuH,HanspalJS, etal. Molecularbasis of spectrin and ankyrin deficiencies in severe hereditary spherocytosis: evidence implicating a primary defect of ankyrin. Blood 1991;77(1):165-73.

79. Cutillo S, Pinto L, Nobili B, Miraglia del Giudice E, et al. Spectrin/band 3 ratio as diagnostic tool in hereditary spherocytosis. Eur J Pediatr 1992;151(1):35-7.

80. Pekrun A, Eber SW, Kuhlmey A, Schröter W. Combined ankyrin and spectrin deficiency in hereditary spherocytosis. Ann Hematol 1993;67(2):89-93.

81. Savvides P,Shalev O, John KM, Lux SE. Combined spectrin and ankyrin deficiency is common in autosomal dominant hereditary spherocytosis. Blood 1993;82(10):2953-60.

82. Mayeur-Rousse C, Gentil M, Botton J, Thibaut MF, et al. Testing for hereditary spherocytosis: a French experience. Haematologica 2012;97(12):e48-9.

83. Stenson PD, Ball EV, Mort M, Phillips AD, et al. Human gene mutation database (HGMD): 2003 update. Hum Mutat 2003;21(6):577-81.

84. Bolton-Maggs PH, Langer JC, Iolascon A, Tittensor P, et al. Guidelines for the diagnosis and management of hereditary spherocytosis-2011 update. Br J Haematol 2012;156(1):37-49. 